\title{
Amplification of MPZL1/PZR promotes tumor cell migration through Src-mediated phosphorylation of cortactin in hepatocellular carcinoma
}

Deshui Jia ${ }^{1,2}$, Ying Jing ${ }^{1,2}$, Zhenfeng Zhang ${ }^{1}$, Li Liu ${ }^{1}$, Jie Ding ${ }^{1}$, Fangyu Zhao ${ }^{1}$, Chao Ge ${ }^{1}$, Qifeng Wang ${ }^{3}$, Taoyang Chen ${ }^{4}$, Ming Yao ${ }^{1}$, Jinjun $\mathrm{Li}^{1}$, Jianren $\mathrm{Gu}^{1}$, Xianghuo $\mathrm{He}^{1}$

${ }^{I}$ State Key Laboratory of Oncogenes and Related Genes, Shanghai Cancer Institute, Renji Hospital, Shanghai Jiao Tong University School of Medicine, Shanghai 200032, China; ${ }^{2}$ Shanghai Medical College, Fudan University, Shanghai 200032, China; ${ }^{3}$ Department of Pathology, Fudan University Shanghai Cancer Center, Shanghai Medical College, Fudan University, Shanghai 200032, China; ${ }^{4}$ Qidong Liver Cancer Institute, Qidong, Jiangsu 226200, China

We have previously identified 1241 regions of somatic copy number alterations (CNAs) in hepatocellular carcinoma (HCC). In the present study, we found that a novel recurrent focal amplicon, 1q24.1-24.2, targets the MPZL1 gene in HCC. Notably, there is a positive correlation between the expression levels of MPZL1 and intrahepatic metastasis of the HCC specimens. MPZL1 can significantly enhance the migratory and metastatic potential of the HCC cells. Moreover, we found that one of the mechanisms by which MPZL1 promotes HCC cell migration is by inducing the phosphorylation and activation of the pro-metastatic protein, cortactin. Additionally, we found that Src kinase mediates the phosphorylation and activation of cortactin induced by MPZL1 overexpression. Taken together, these findings suggest that MPZL1 is a novel pro-metastatic gene targeted by a recurrent region of copy number amplification at 1q24.1-24.2 in HCC.

Keywords: copy number alterations; MPZL1; cortactin; migration; hepatocellular carcinoma

Cell Research (2014) 24:204-217. doi:10.1038/cr.2013.158; published online 3 December 2013

\section{Introduction}

Hepatocellular carcinoma (HCC) is one of the tumor types with worst prognosis worldwide. Cancer metastases, both intrahepatic and extrahepatic, are major factors causing the mortality of HCC patients. Recently, a number of cancer genes have been reported to be associated with the tumor metastasis in $\mathrm{HCC}$, including $C L U$, KAI1, ROCK2 and TWIST [1-4]. Furthermore, we have previously identified several microRNAs that play critical roles in HCC metastasis, such as miR-30D, miR-151 and miR-210 [5-7]. Additionally, many signaling pathways have also been implicated in the process of HCC metastasis, including RAF/MEK/ERK pathway, WNT/

Corresponsdence: Xianghuo $\mathrm{He}$

Tel/Fax: 86-21-64436539

E-mail: xhhe@shsci.org

Received 9 May 2013; revised 10 September 2013; accepted 25 September 2013; published online 3 December 2013 $\beta$-catenin pathway, insulin-like growth factor pathway, hepatocyte growth factor/c-MET pathway and growth factor-regulated angiogenic signaling [8]. However, the molecular mechanisms underlying HCC metastasis remain greatly elusive. Thus, efforts to understand the genetic basis and molecular mechanism of initiation and progression of this lethal disease will help to save life of HCC patients.

An effective method of identifying driver genes with causal roles in carcinogenesis is the detection of genomic regions that undergo frequent alterations in cancers. Chromosomal copy number alterations (CNAs) can lead to the activation of oncogenes and the inactivation of tumor suppressors in human cancers [9]. Although certain regions of recurrent CNAs harbor a single gene, most of these regions include several genes; thus, combining structural and functional genomics studies is of vital importance [10]. For example, the identification of CDK8 as an essential gene in colon cancers harboring amplifications in a 16-gene region of chromosome 13 led 
to a deeper investigation of the essentiality of all of the genes in this region. The suppression of each gene in this region by RNAi showed that $\mathrm{CDK} 8$ was the only one required for the survival of colon cancer cells with copy number amplification of chromosome 13 [11]. To identify recurrent regions of CNAs in $\mathrm{HCC}$, we recently performed a genome-wide copy number analysis of 58 pairs of HCC primary tumors and adjacent non-tumor tissues using the Affymetrix SNP 6.0 array, from which a total of 1241 regions of somatic CNAs were derived [12]. Based on the hypothesis that genes within smaller amplicons are more likely to be tumor-promoting than those from larger chromosomal alterations [13], a number of subtle CNAs $(\leq 3 \mathrm{Mb})$ were further uncovered among these regions, including regions of copy number amplification at 1q21.2-21.3 and 1q24.1-24.2. Copy number gain at $1 \mathrm{q}$ is one of the most frequently detected alterations in HCC (58\% - 78\%) and has been suggested to be an early genomic event in the process of HCC development [14]. Of note, the 1q21 amplicon has already been identified and well documented in $\operatorname{HCC}[15,16]$, whereas to date, the 1q24 amplicon has rarely been indicated or studied in HCC.

In this study, we take steps to explore and characterize the potential cancer genes targeted by this focal recurrent amplicon in HCC. By integrated analysis of copy number and expression profiling data, we found that the recurrent region of the 1q24.1-24.2 amplicon specifically targets the MPZL1 gene in HCC. MPZL1, also known as PZR, is a cell surface glycoprotein belonging to the immunoglobulin superfamily and, in the human, consists of three isoforms differing in their cytoplasmic sequences [17]. Recent studies have shown that MPZL1 could promote the fibronectin-dependent migration of murine mesenchymal-derived MEF cells $[17,18]$, and may be involved in adhesion-dependent signaling $[19,20]$. However, the functional roles and clinical implications of MPZL1 amplification and overexpression in human cancers are largely unknown. In the current study, we showed that MPZL1 plays a pivotal role in human cancer cell migration and tumor metastasis. Importantly, overexpression of MPZL1 was identified to be associated with the intrahepatic metastasis of HCC patients. Our results revealed that the pro-metastatic function of MPZL1 might be through promoting Src kinase-mediated phosphorylation and activation of cortactin to increase cell migration.

\section{Results}

Recurrent genomic amplification of 1q24.1-24.2 targets MPZL1 in HCC

It has long been thought that DNA CNAs frequently contribute to tumor initiation and progression. To explore this, we followed up with our previous studies in which Affymetrix single-nucleotide polymorphism 6.0 arrays were used to identify novel regions of amplification and deletion in human HCC specimens [12]. Among the 1 241 regions of somatic CNAs identified in $\mathrm{HCC}$, we uncovered a novel recurrent region of focal amplification (1q24.1-24.2) with a frequency of $44.8 \%(26 / 58)$ in HCC. To further identify the potential driver genes located in this region, we mainly focused on differentially expressed genes within this region for further studies by the integrated analysis of copy number and expression profiling data [12], from which four upregulated genes were identified in the broad region of 1q24 copy number gain, including $M P Z L 1$ (also termed PZR), NME7 and $A T P 1 B 1$ at $1 \mathrm{q} 24.2$ and $P I G C$ at $1 \mathrm{q} 24.3$ (Figure $1 \mathrm{~A}$ and Supplementary information, Table S1). Furthermore, both the DNA dosages and expression levels of these genes were confirmed by quantitative real-time PCR (qPCR) in an independent cohort of HCC specimens. However, only the MPZL1 gene could be confirmed at both DNA dosage and mRNA expression level (Figure 1B, 1C and Supplementary information, Figure S1). Additionally, the positive correlation between the DNA dosage and expression level of the MPZL1 gene was also confirmed (Figure 1D). Therefore, these data suggested that the MPZL1 gene is one of the candidate cancer genes targeted by the recurrent genomic amplification of 1q24.124.2 , and it was selected for further study to explore its biological function and molecular mechanism.

The expression levels of MPZL1 correlate with the malignant features of $\mathrm{HCC}$

The human MPZL1 consists of three isoforms (MPZL1, $M P Z L 1 a$ and MPZL1b) differing in their cytoplasmic sequences [17]. We examined the relative expression levels of three isoforms of MPZL1 in 58 pairs of HCC and adjacent non-tumor tissues by q-PCR. We found that only the isoform a (MPZL1) is overexpressed in $\mathrm{HCC}$ compared with adjacent non-tumor tissues (Supplementary information, Figure S2). Consistent with our findings, Oncomine expression analysis also revealed higher MPZL1 expression in HCC tissues than in normal liver tissues in two independent sets of HCC specimens [21, 22] (Supplementary information, Figure S3).

Based on the relative expression levels of the MPZL1 gene in 58 pairs of $\mathrm{HCC}$ primary tumor and adjacent non-tumor tissues, we undertook the analysis of the clinical significance of MPZL1 gene overexpression in HCC. First, by comparison of the relative MPZL1 expression levels between the paired primary tumor and adjacent non-tumor tissues, we found that the proportion of $\mathrm{HCC}$ 
A

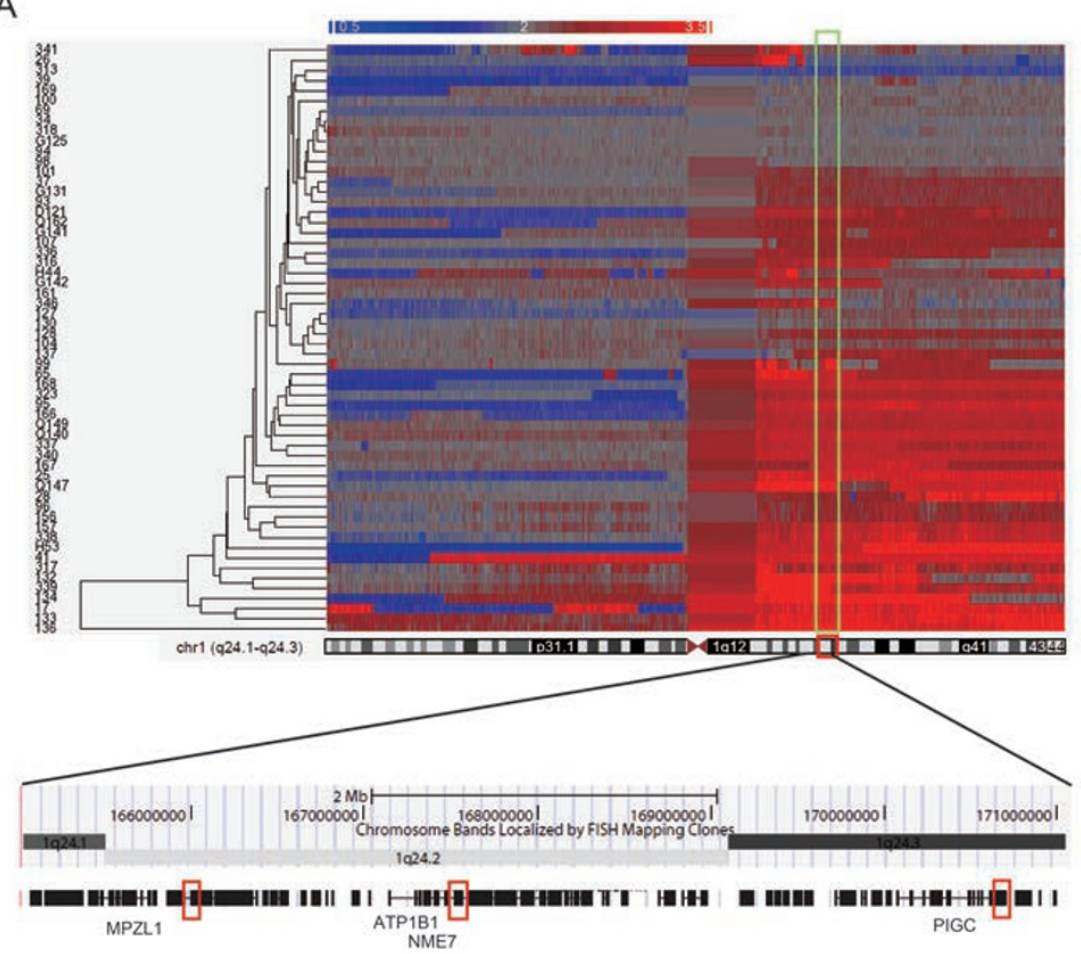

B

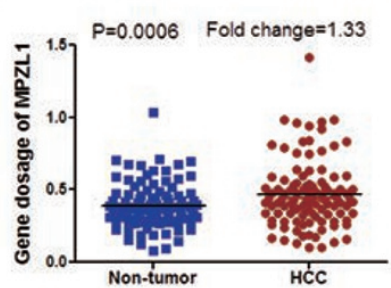

C

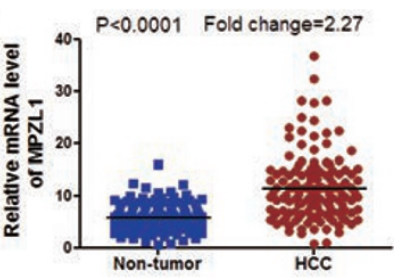

D

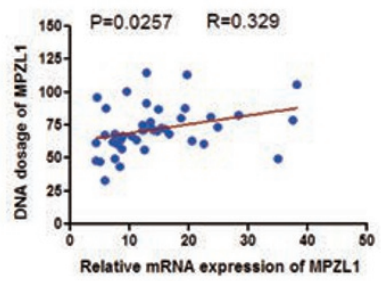

Figure $1 \mathrm{~A}$ recurrent region of amplification at 1q24.1-24.2 targets the MPZL1 gene in HCC. (A) A schematic diagram of the 1q24.1-24.2 amplicon and four upregulated genes (MPZL1, ATP1B1, NME7 and PIGC) located in the broad region of 1q24. The upper panel displays an unsupervised hierarchical clustering of copy number data from $58 \mathrm{HCC}$ specimens, and tumors are ordered from top to bottom according to the hierarchical clustering of CNAs for chromosome 1. Red, copy number gains; blue, copy number losses. (B) Validation of DNA dosages of the MPZL1 by q-PCR in an independent cohort of 125 paired HCCs and adjacent non-tumor tissues with the genomic DNA. (C) Confirmation of gene expression of MPZL1 by q-PCR in an independent cohort of 130 paired HCCs and adjacent non-tumor tissues with cDNA. (D) Correlation analysis of DNA dosage and mRNA expression for the MPZL1 gene in 46 HCC primary tissues with both genomic DNA and CDNA. The correlation was analyzed by the two-tailed Pearson Correlation Test.

specimens with MPZL1 upregulation (43.1\%) was much higher than that with MPZL1 downregulation (12.1\%) (Figure 2A). Importantly, there was a positive correlation between the expression levels of MPZL1 and intrahepatic metastasis of the HCC specimens (Supplementary information, Table S2). Moreover, according to the results of q-PCR analysis of the relative expression levels of MPZL1 gene in $58 \mathrm{HCC}$ primary tissues, the expression levels of the MPZL1 gene in the HCC primary tumors with intrahepatic metastasis significantly increased compared with those without intrahepatic metastasis (Figure 2B), whereas the expression levels of the MPZL1 gene in the high grade HCC primary tumors were significantly higher than those in the low-grade ones (Figure 2C). Together, these results implied that the increased expression levels of the MPZL1 gene may be associated with the malignant progression and metastasis of $\mathrm{HCC}$, thus providing clues to further explore its biological function and molecular mechanism in HCC progression.
MPZL1 increases the migratory and metastatic potential of HCC cells

To choose suitable cellular models to study the biological function of $M P Z L 1$, we first examined the relative protein levels of the MPZL1 gene in six HCC cell lines. The results showed that different expression levels of the MPZL1 gene can be detected in all the six HCC cell lines (Figure 3A). Specifically, the protein level of MPZL1 was relatively low in HepG2, Hep3B, HUH-7 and SMMC-7721 cells, which have no or low metastatic potential [4, 23-25], whereas the protein level of the MPZL1 gene was relatively high in SK-HEP-1 and Li-7 cells, which have high metastatic potential $[24,26]$.

First, we stably knocked down the expression of the MPZL1 gene through lentiviral infection in two highly metastatic cell lines, SK-HEP-1 and Li-7; the interference efficiency was confirmed by immunoblotting (Figure 3B and Supplementary information, Figure S4). Subsequently, the effect of RNA interference-mediated 
A

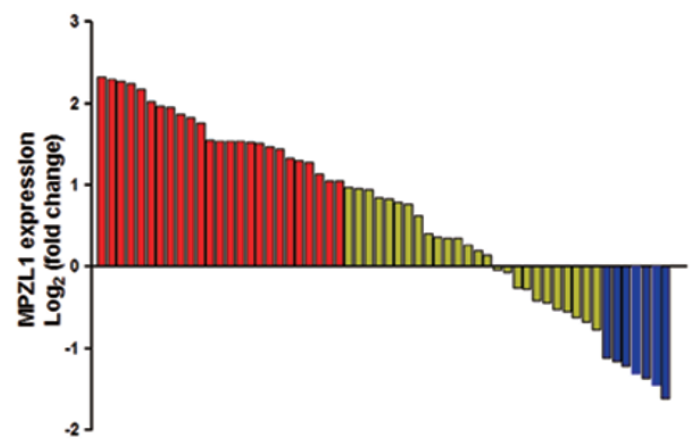

B

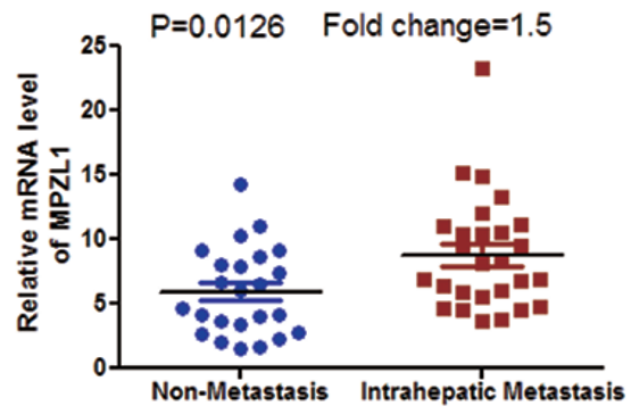

Downregulation

$12.1 \%$

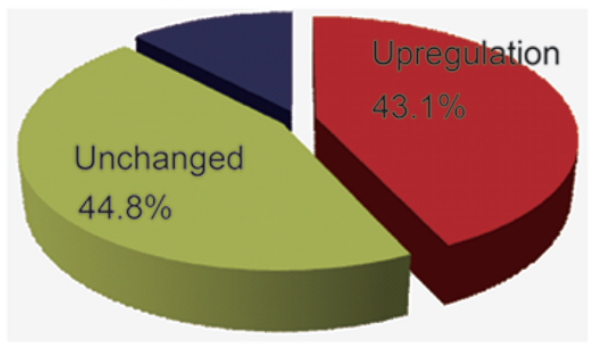

C

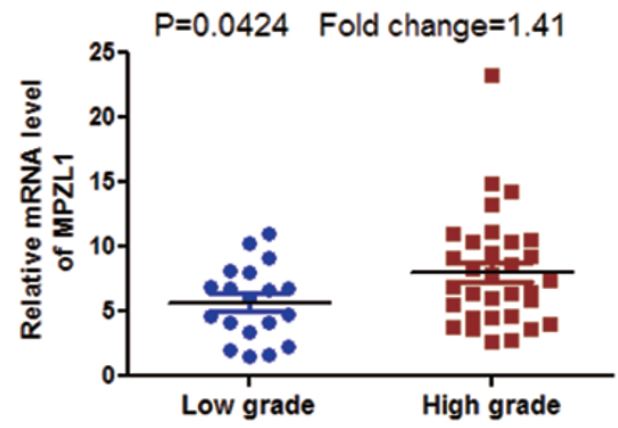

Figure 2 Overexpression of MPZL1 correlates with malignant features of HCC. (A) The expression levels of MPZL1 in 58 paired HCC and matched non-tumor tissues were determined by q-PCR. The data are expressed as the log fold $_{2}$ change $\left(\triangle \mathrm{Ct}\left[\mathrm{HCC} /\right.\right.$ Non.]). Significant upregulation of MPZL1 expression in paired HCC/non-tumor samples was defined as a log ${ }_{2}$ fold change $>1$ (i.e.,2-fold). The pie chart shows the proportions of HCC samples showing upregulation (red), downregulation (blue), and no change (yellow). (B) Overexpression of MPZL1 in primary HCCs with intrahepatic metastasis compared with that without intrahepatic metastasis according to the results of q-PCR. (C) Overexpression of MPZL1 in primary HCCs in high grade (grade $=3$ and 4 ) compared with that in low grade (grade $=1$ and 2 ) according to the results of q-PCR. Statistical analysis of differences between the two groups was performed by unpaired Student's $t$-test and $P<0.05$ was considered statistically significant.

knockdown of the MPZL1 gene on the proliferation of these cells was determined by CCK- 8 assays; the results showed that disruption of MPZL1 gene expression had no significant effect on the proliferation of HCC cells (Figure 3C and Supplementary information, Figure S4). Furthermore, we found that knockdown of MPZL1 gene expression inhibited the migratory and invasive abilities of HCC cells in transwell assays (Figure 3D, 3E and Supplementary information, Figure S4).

To further verify the role of MPZL1 in the HCC cell migration, the MPZL1 gene was overexpressed by lentiviral infection in HUH-7 and SMMC-7721 cells, which was confirmed by immunoblotting (Figure 4A). Subsequently, we determined the effect of MPZL1 overexpression on the proliferation of these HCC cells both in vitro and in vivo. The results showed that exogenous overexpression of the MPZL1 gene has no significant effect on HCC cell proliferation (Figure 4B and Supplementary information, Figure S5). Furthermore, we examined the effect of MPZL1 overexpression on the migratory and invasive abilities of these HCC cells by transwell assays, and the results showed that ectopic expression of MPZL1 significantly promoted the in vitro migration and invasion of HCC cells (Figure 4C and 4D). Finally, we found that MPZL1 could promote the in vivo metastasis of $\mathrm{HCC}$ cells in mouse models by intravenous injection of tumor cells into mouse (Figure 4E and 4F). Taken together, these results suggested that the MPZL1 gene may play an important role in the cell migration and tumor metastasis of HCC.

Ectopic expression of MPZL1 leads to increased phosphorylation of multiple pro-metastatic proteins

We performed a phospho-proteomics-based study using a phospho-antibody microarray to explore the molecular mechanisms underlying MPZL1-mediated 
A

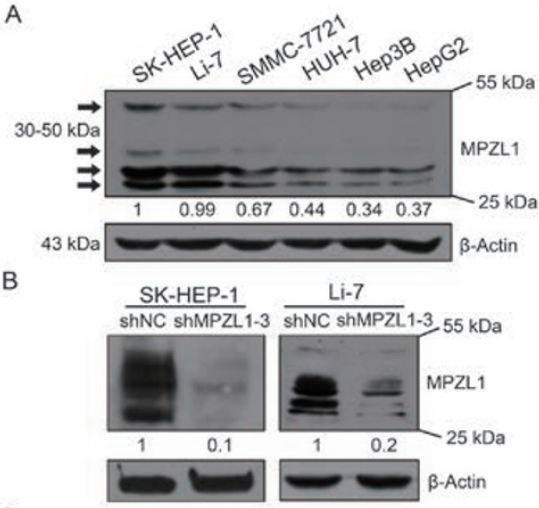

C
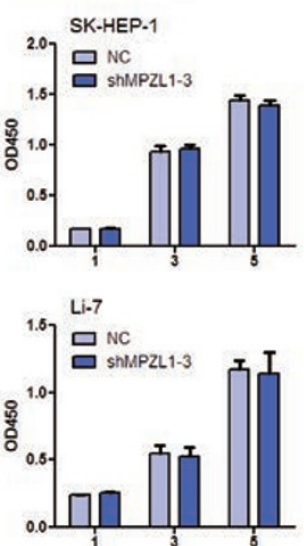
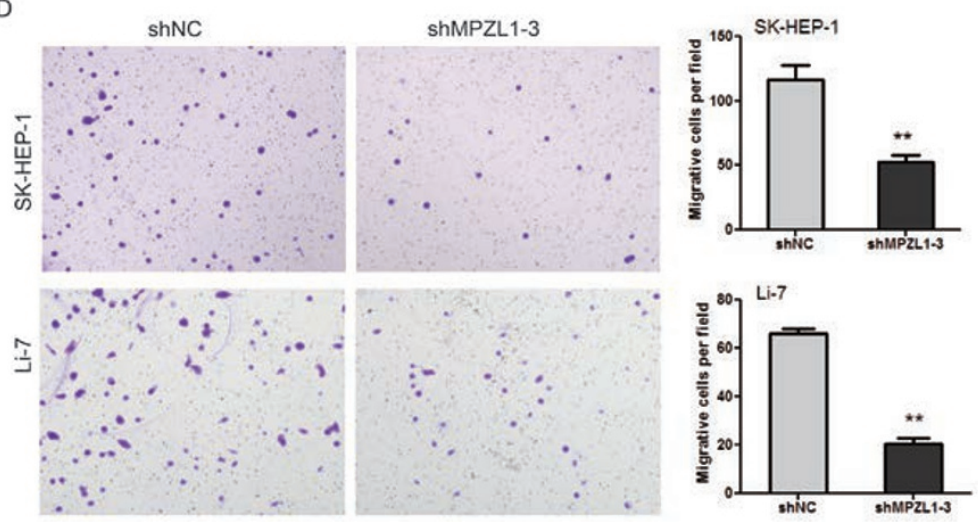

E
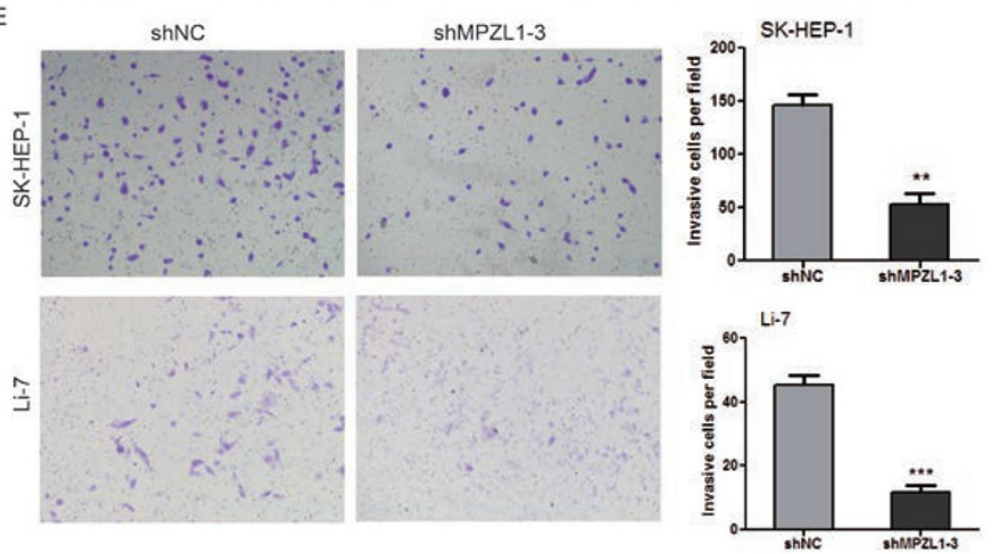

Figure 3 Targeted downregulation of MPZL1 attenuates HCC cell migration. (A) The detection of the relative protein levels of MPZL1 in six HCC cell lines by immunoblotting. Arrows denote the position of MPZL1. (B) The detection of shRNA-mediated knockdown of MPZL1 in SK-HEP-1 and Li-7 cells by immunoblotting. (C) A representative result of the CCK-8 assays for the effects of MPZL1 knockdown on the in vitro proliferation of SK-HEP-1 and Li-7 cells. (D, E) Representative results of the transwell assays for the effects of MPZL1 knockdown on the in vitro migratory and invasive abilities of SK-HEP-1 and Li-7 cells, respectively. Statistical analysis of differences between groups was performed by unpaired Student's $t$-test. The results are shown as the mean \pm s.e.m, ${ }^{* *} P<0.01 ;{ }^{* * *} P<0.001$.

cell migration and tumor metastasis [27]. The microarray provides a high-throughput platform for an efficient protein phosphorylation status profiling, with detection and analysis of phosphorylation events at specific sites to identify MPZL1 downstream effectors that can regulate metastasis. Using cell lysates derived from HUH7-VECTOR and HUH-7-MPZL1 cells, we identified a spectrum of proteins of which the phosphorylation levels were increased by $>15 \%$ in HUH-7-MPZL1 cells relative to HUH-7-VECTOR cells. Many of these proteins, when phosphorylated, are important for cell migration and tumor metastasis. These pro-metastatic proteins included focal adhesion kinase (FAK) [28 ], Src [29 ] and cortactin [30] (Supplementary information, Tables S3 and S4).

We further confirmed, by immunoblotting, that stable overexpression of MPZL1 resulted in increased phosphorylation of FAK and Src in both HUH-7 and Hep3B cells (Figure 5A). Additionally, targeted knockdown of the MPZL1 gene led to a reduced phosphorylation of these two proteins in SK-HEP-1 cells (Figure 5B). Although MPZL1, as a membrane protein, is unlikely to directly regulate the activation of these pro-metastatic tyrosine kinases by phosphorylation, the increased activation of FAK and Src upon MPZL1 overexpression indicates the reprogramming of the HCC pro-metastatic signaling network upon MPZL1 overexpression. Recently, many studies have documented a role for the cortactin in promoting cell motility and metastasis [31, 32]. Moreover, cortactin was originally identified as a substrate for the Src family kinases, which are major kinases in the tyrosine phosphorylation of cortactin [33]. In addition, MPZL1 has also been shown to activate Src kinase upon stimulation of cells with extracellular stimuli [34]. However, the roles of MPZL1 in the phosphorylation and acti- 
A

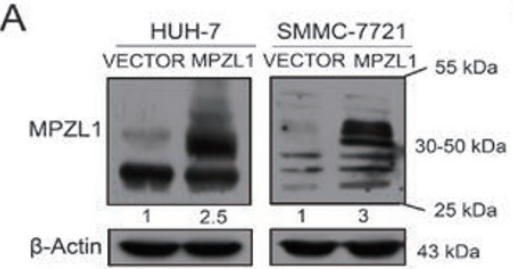

B
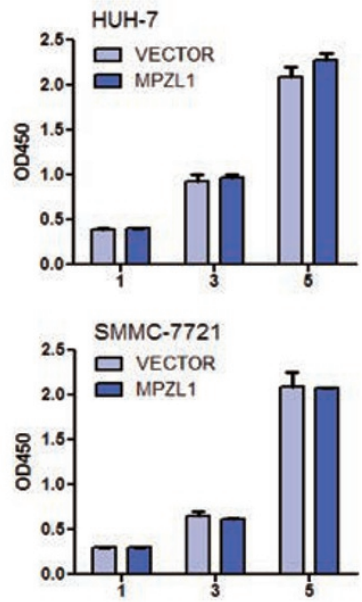

$\mathrm{E}$

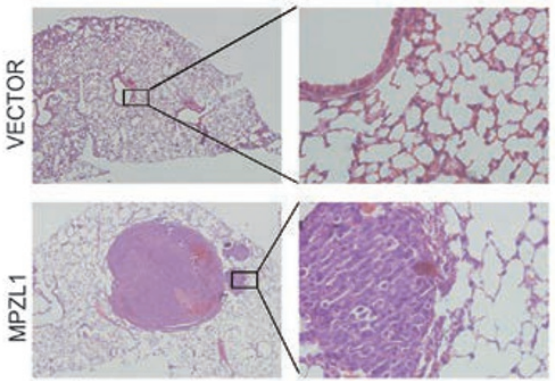

C

D
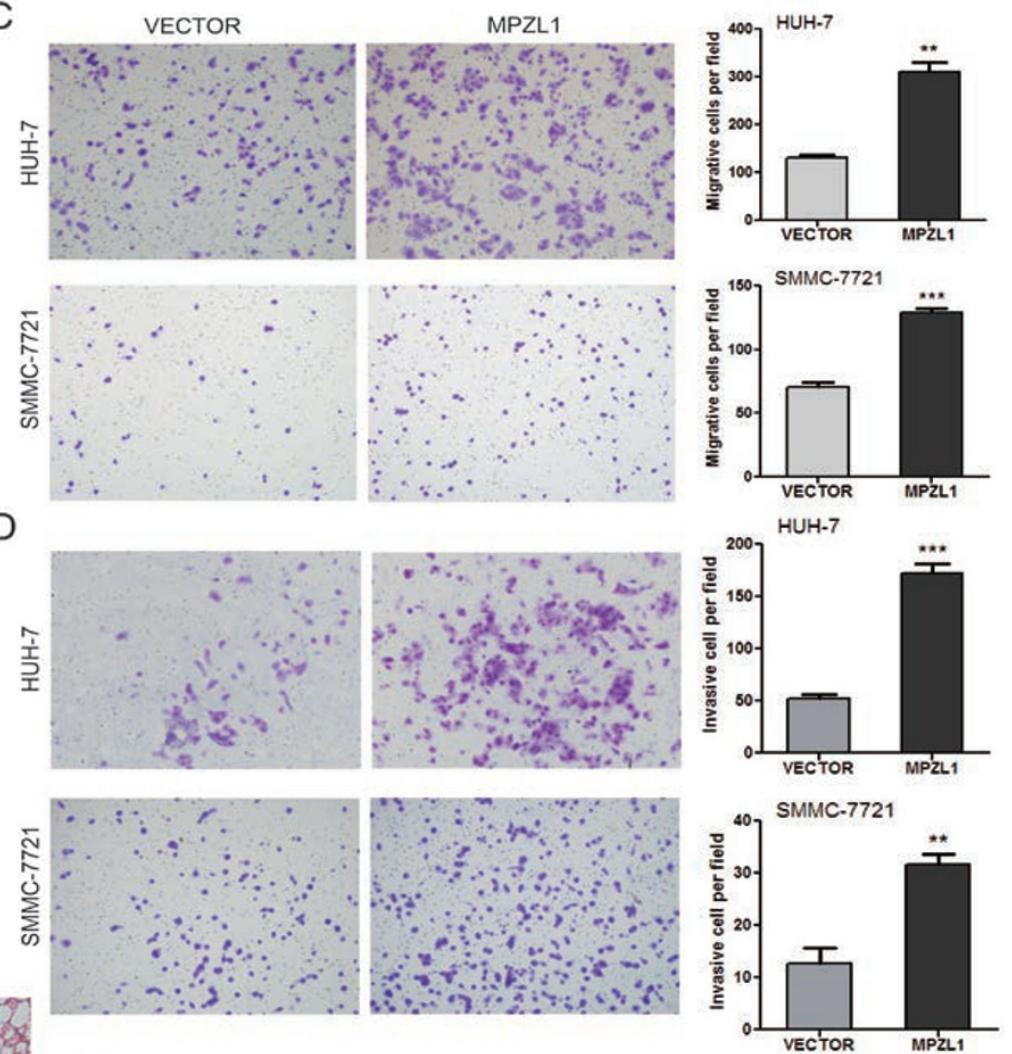

$\mathrm{F}$

Incidence of lung metastasis in mouse models.

\begin{tabular}{lcc}
\hline Group & Microscopic metastasis & $p$ value \\
\hline HUH-7 VECTOR & $0 / 7$ & 0.005 \\
HUH-7 MPZL1 & $6 / 7$ & \\
\hline$p$ value represents the probability from $\times 2$ test for the incidence of \\
lung metastasis between two groups.
\end{tabular}

Figure 4 Overexpression of MPZL1 promotes HCC cell migration and metastasis. (A) The detection of lentivirus-mediated overexpression of MPZL1 in HUH-7 and SMMC-7721 cells by immunoblotting. (B) A representative result of the CCK-8 assays for the effects of MPZL1 overexpression on the in vitro proliferation of $\mathrm{HUH}-7$ and SMMC-7721 cells. (C, D) Representative results of the transwell assays for the effects of MPZL1 overexpression on the in vitro migratory and invasive abilities of HUH-7 and SMMC-7721 cells, respectively (unpaired Student's $t$-test, the mean \pm s.e.m, ${ }^{* *} P<0.01$; ${ }^{* * *} P<0.001$ ). (E, F) The effects of MPZL1 overexpression on the in vivo metastatic abilities of $\mathrm{HUH}-7$ cells in xenograft models of nude mice $(n=7)$. Representative results of histological examination of mouse lungs for metastatic nodules in two groups, HUH-7 VECTOR and HUH-7 MPZL1 (E) and the incidence of lung metastasis in the two groups of the mouse models (F). Original magnifications, $40 \times$ (inset, 200x). Statistical analysis of differences between groups was performed by the $X^{2}$ test and $P<0.05$ was considered statistically significant.

vation of cortactin have not been determined. Therefore, we propose that a novel MPZL1/Src/cortactin signaling cascade exists and functions in the process of HCC cell migration. Subsequently, we confirmed the increased phosphorylation of cortactin in HUH-7 and Hep3B cells upon MPZL1 overexpression by immunoblotting (Figure 5C). Moreover, targeted knockdown of the MPZL1 gene by shRNA also resulted in reduced phosphorylation of the cortactin in SK-HEP-1 cells (Figure 5D).

MPZL1 was previously identified as a SHP-2-binding partner in epithelial cells, and the intracellular portion of MPZL1 has two immunoreceptor tyrosine-based inhibition motifs that specifically interact with SHP-2, an SH2 domain-containing tyrosine phosphatase with a crucial role in cell signaling $[18,19]$. Recently, it has been reported that the SHP-2 tyrosine phosphatase activates 
A

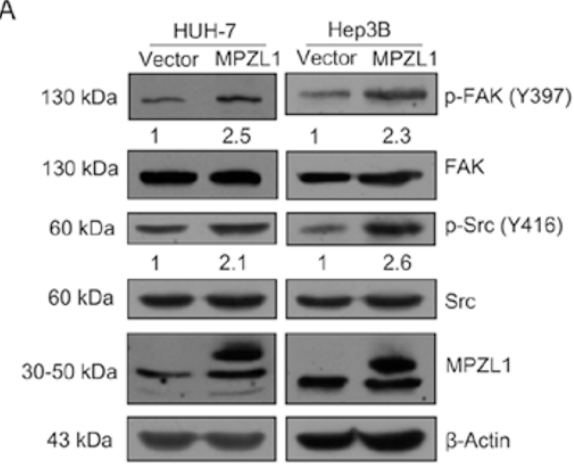

C

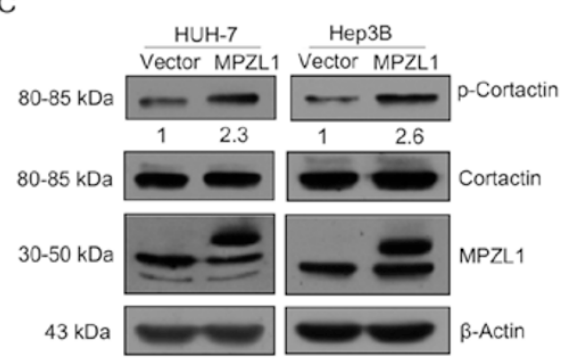

B

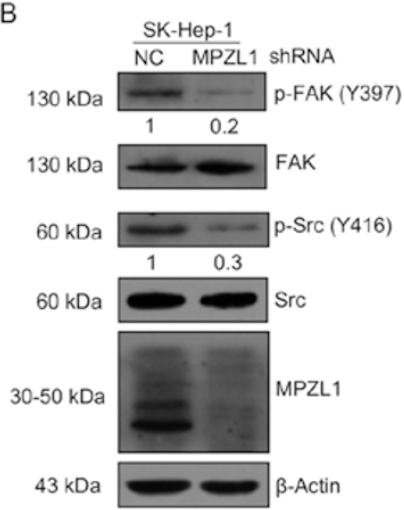

D
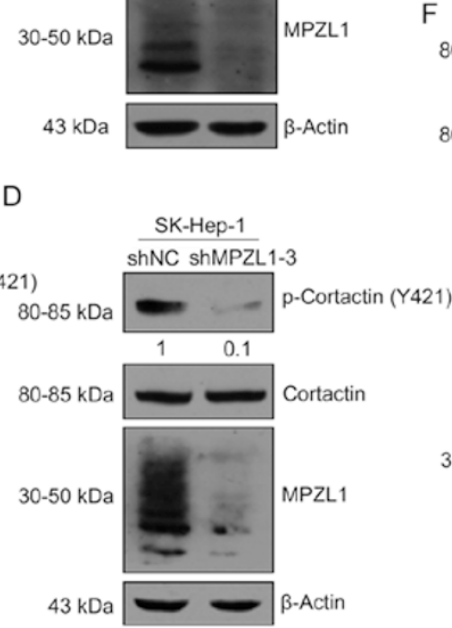
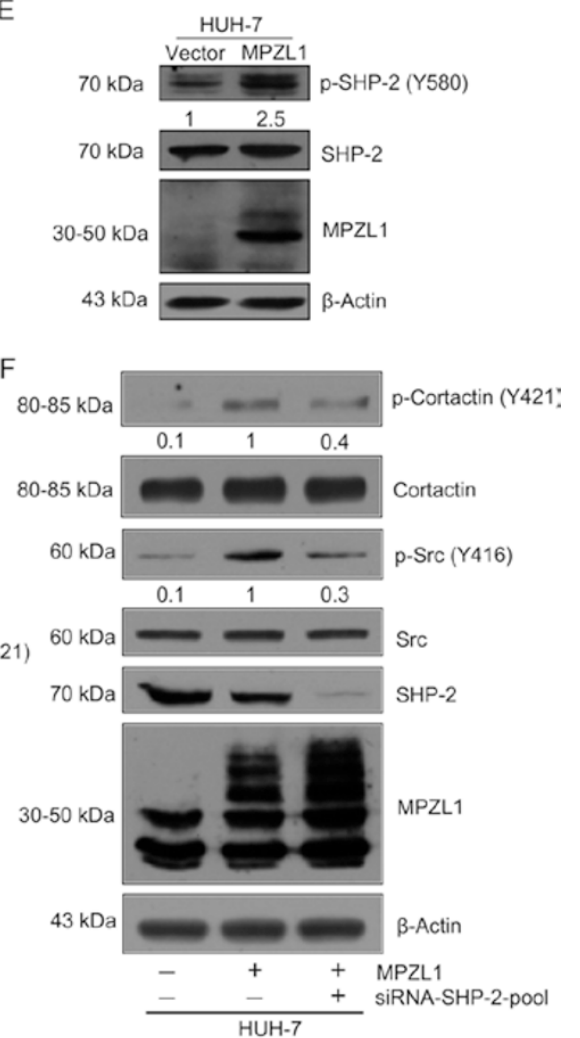

Figure 5 Ectopic expression of MPZL1 leads to increased phosphorylation of multiple pro-metastatic proteins. (A) The overexpression of MPZL1 in HUH-7 and Hep3B cells increases the phosphorylation of FAK (Y397) and Src (Y416) proteins. (B) The knockdown of MPZL1 in SK-HEP-1 cells decreases the phosphorylation of FAK (Y397) and Src (Y416) proteins. (C) The overexpression of MPZL1 in HUH-7 and Hep3B cells leads to the increased phosphorylation of cortactin (Y421) protein. (D) The knockdown of MPZL1 in SK-HEP-1 cells decreases the phosphorylation of cortactin (Y421) protein. (E) The overexpression of MPZL1 in HUH-7 cells results in the increased phosphorylation of SHP-2 (Y580) protein. (F) Targeted knockdown of the SHP-2 gene using siRNAs attenuates the increased phosphorylation of Src (Y416) and cortactin (Y421) caused by the overexpression of MPZL1 in HUH-7 cells.

the Src tyrosine kinase via a non-enzymatic mechanism [35]. We found that overexpression of MPZL1 resulted in increased phosphorylation of SHP2 (Figure 5E and Supplementary information, Table S3). Furthermore, we found that siRNA-mediated knockdown of the SHP-2 gene significantly attenuated the increased phosphorylation of the active form of Src (Y416) and cortactin (Y421) caused by overexpression of MPZL1 in HUH-7 cells (Figure 5F). This result suggested that the activation of Src kinase by overexpression of MPZL1 is probably associated with the recruitment and activation of SHP-2.

\section{MPZL1 promotes cancer cell migration through phos-} phorylation and activation of cortactin

To further characterize the signaling properties of $M P Z L 1$ in cancer cell migration, we focused on the prometastatic protein, cortactin. To clarify whether phos- phorylation of cortactin is involved in MPZL1-mediated cancer cell migration, we determined the effect of RNAimediated knockdown of the CTTN gene, which encodes cortactin, on the migration of the MPZL1-expressing metastatic SK-HEP-1 cells. The CTTN shRNA was specific in decreasing cortactin protein expression in SKHEP-1 cells (Supplementary information, Figure S6A). Stable knockdown of the CTTN gene in SK-HEP-1 cells resulted in a significant reduction of their in vitro migratory and invasive abilities but did not significantly affect their proliferation (Supplementary information, Figure S6B-S6D).

We further demonstrated that enforced expression of MPZL1 could enhance the migratory ability of HUH7 cells, while shRNA-mediated knockdown of the CTTN gene significantly attenuated the migration of these MPZL1-overexpressing cells (Figure 6A). More- 


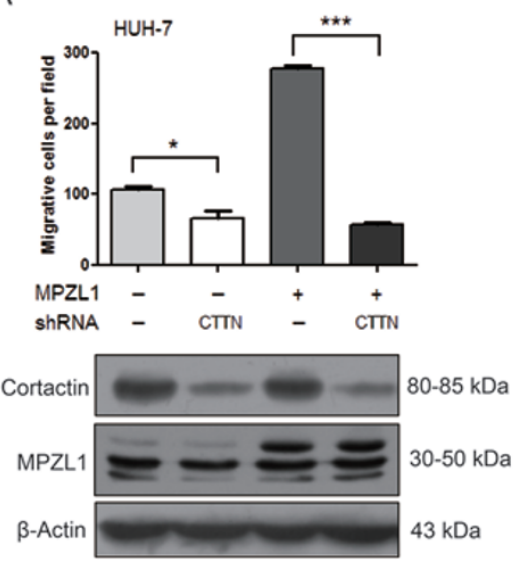

D

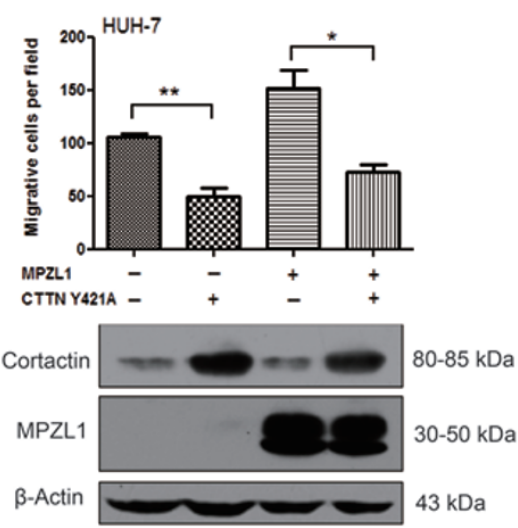

B
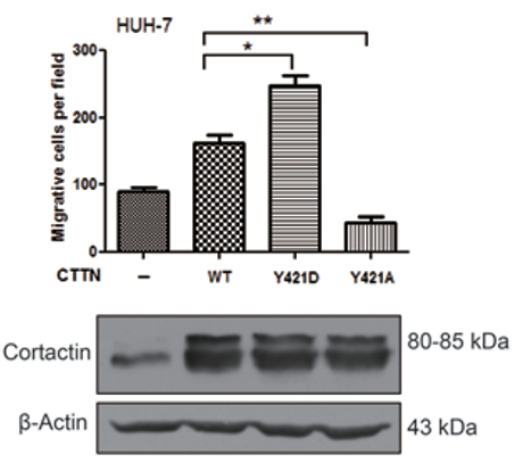

$\mathrm{E}$

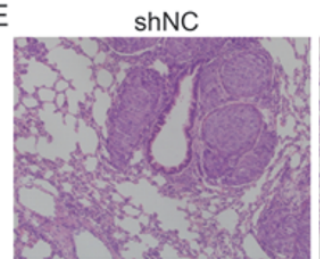

shMPZL1
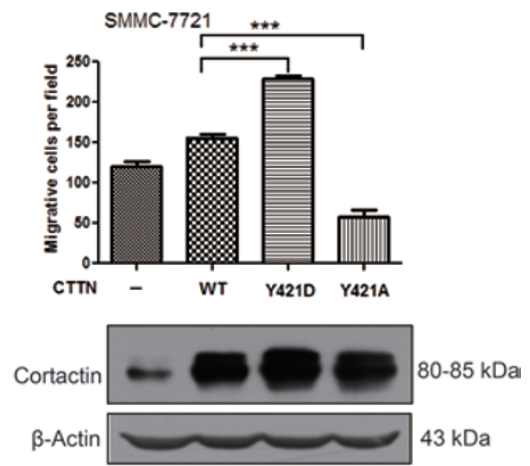

$\mathrm{F}$

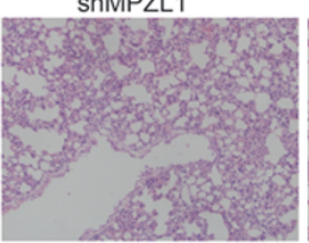

shMPZL1 + CTTN Y421D

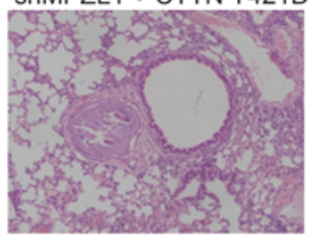

Incidence of lung metastasis in mouse models

\begin{tabular}{lcc}
\hline Groups & Micrometastasis & p value \\
\hline shNC & $3 / 10$ & \\
shMPZL1 & $0 / 10$ & 0.039 \\
shMPZL1 + CTTN Y421D & $5 / 10$ & \\
\hline
\end{tabular}

$p$ value represents the probability of from $\mathrm{X} 2$ test for the incidence of lung metastasis among three groups.

Figure 6 MPZL1 promotes the metastatic potential of HCC cells through the phosphorylation and activation of cortactin. (A) The overexpression of MPZL1 promoted the migratory ability of $\mathrm{HUH}-7$ cells, while the targeted downregulation of CTTN significantly attenuated MPZL1-induced cell migration (unpaired Student's $t$-test, the mean \pm s.e.m, ${ }^{*} P<0.05$; ${ }^{* * *} P<0.001$ ). (B, C) Stable expression of the phospho-mimetic CTTN Y421D mutant, but not the phospho-deficient CTTN Y421A mutant, further significantly enhanced cell migration of HUH-7 (B) and SMMC-7721 (C) compared with that of CTTN WT (one-way ANOVA analysis, the mean \pm s.e.m, ${ }^{*} P<0.05$; ${ }^{* *} P<0.01$; ${ }^{* *} P<0.001$ ). (D) The overexpression of the phospho-deficient CTTN Y421A mutant significantly attenuated MPZL1-induced cell migration (unpaired Student's $t$-test, the mean \pm s.e.m, ${ }^{*} P<$ 0.05; $\left.{ }^{*} P<0.01\right)$. (E, F) The effects of phospho-mimetic CTTN Y421D mutant on the in vivo metastatic abilities of HCCLM3shMPZL1 cells in xenograft models of nude mice $(n=10)$. Representative results of histological examination of mouse lungs for metastatic nodules in three groups, shNC, shMPZL1 and shMPZL1 + CTTN Y421D (E) and the incidence of lung metastasis in the three groups of the mouse models (F). Original magnifications, 200×. Statistical analysis of differences among groups was performed by the $\chi^{2}$ test and $P<0.05$ was considered statistically significant.

over, stable expression of the CTTN wild-type construct (CTTN WT) resulted in significantly increased migration of HUH-7 and SMMC-7721 cells, and cells expressing a phospho-mimetic mutant, CTTN Y421D, showed a further increase in the migratory ability (Figure 6B and 6C). In contrast, overexpression of a phospho-deficient mutant, CTTN Y421A, resulted in decreased cell migration of HUH-7 and SMMC-7721 cells (Figure 6B and 6C). In addition, overexpression of CTTN Y421A mutant could abrogate the cell migration induced by MPZL1 overexpression in HUH-7 cells (Figure 6D). Finally, we examined the effects of overexpression of CTTN Y421D mutant in MPZL1-knockdown cells on tumor metastasis of HCC cells in mouse models. The results showed that knockdown of MPZL1 could inhibit the lung metastasis of HCCLM3 cells, whereas overexpression of the phospho-mimetic CTTN Y421D mutant could rescue the lung metastasis in MPZL1-knockdown cells (Figure 6E and $6 \mathrm{~F})$. Taken together, these findings suggested that one of the mechanisms by which MPZL1 promotes the migration and metastasis of HCC cells is through the induction of the phosphorylation and activation of cortactin. 
A

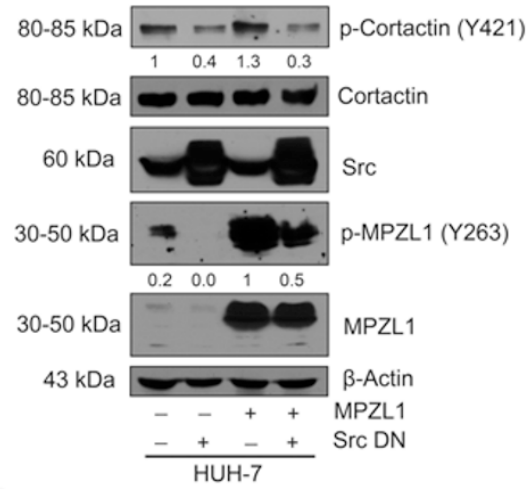

D

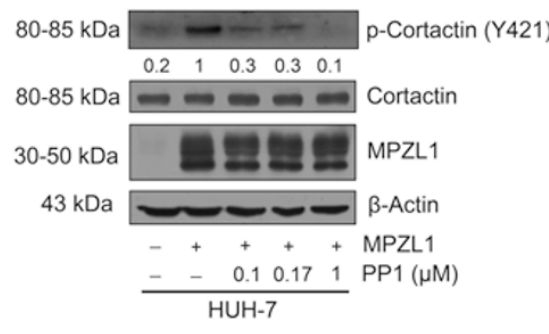

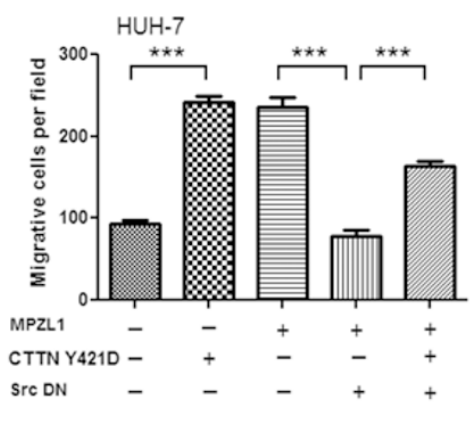

E

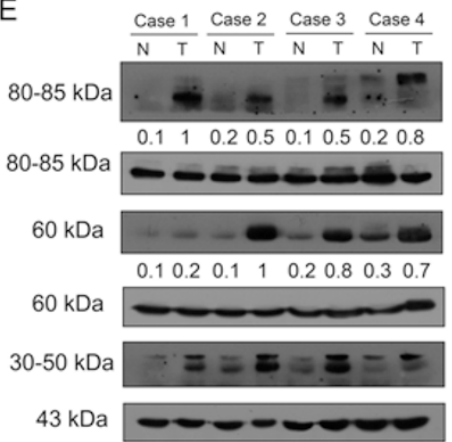

C

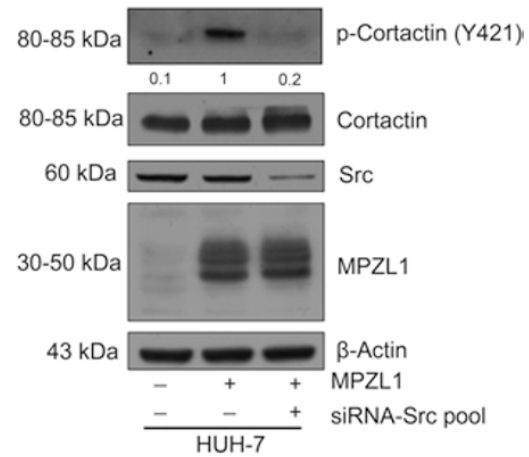

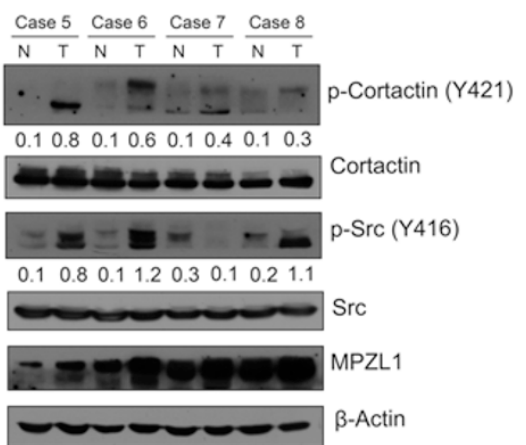

Figure 7 Src kinase mediates the phosphorylation of cortactin induced by MPZL1 overexpression. (A) Stable expression of the dominant-negative mutant of Src kinase (K298M/Y530F kinase dead mutant with open conformation, Src DN) attenuates the phosphorylation of cortactin induced by MPZL1 overexpression in HUH-7 cells. (B) Stable expression of the Src DN mutant significantly inhibits the migration of $\mathrm{HUH}-7$ cells induced by MPZL1 overexpression, which can be restored by the overexpression of a constitutively active mutant of cortactin, CTTN Y421D (unpaired Student's $t$-test, the mean \pm s.e.m, ${ }^{* *} P<0.01$ ). (C) siRNA-mediated knockdown of Src attenuates the increased phosphorylation of cortactin (Y421) induced by MPZL1 overexpression in HUH-7 cells. (D) A Src kinase inhibitor (PP1) attenuates the phosphorylation of cortactin induced by MPZL1 overexpression in $\mathrm{HUH}-7$ cells. Treatment of HUH-7-MPZL1 cells with different concentration of PP1 $(0.1,0.17$ and $1 \mu \mathrm{M})$ could attenuate the increased phosphorylation of cortactin induced by MPZL1 overexpression. (E) The examination of the effects of MPZL1 overexpression on the phosphorylation of cortactin (Y421) and Src (Y416) in eight cases of paired human HCC primary tumor and adjacent non-tumor tissues by immunoblotting ( $\mathrm{N}$, non-tumor; T, primary tumor).

Src kinase mediates the phosphorylation and activation of cortactin induced by MPZL1 in HCC cells

According to the results of the phospho-antibody array and immunoblotting, the phosphorylation level of an active form of Src (phospho-tyrosine 416) was significantly increased upon MPZL1 overexpression (Figure 5B). Moreover, we also found that the phosphorylation level of negative regulatory site of Src (Y527) was significantly decreased in MPZL1-overexpressing cells (Supplementary information, Figure S7). These data suggested that the activation of Src kinase may be responsible for the tyrosine phosphorylation of cortactin in HCC cells. To determine whether the Src kinase activity is required for MPZL1-induced phosphorylation of cortactin, we first expressed a dominant-negative Src mutant (Src K298M/ Y530F) [36] in HUH-7-MPZL1 cells. The dominant- negative Src mutant could inhibit the phosphorylation of cortactin (Figure 7A) and reduced the migration of HUH-7-MPZL1 cells (Figure 7B). Moreover, stable overexpression of the phospho-mimetic CTTN Y421D mutant was able to reverse the inhibitory effect of the Src dominant-negative mutant on the migratory ability of HUH-7-MPZL1 cells (Figure 7B). Furthermore, targeted knockdown of the $\operatorname{Src}$ gene by siRNA also resulted in reduced phosphorylation of cortactin in HUH-7-MPZL1 cells (Figure 7C). In addition, we treated HUH-7-MPZL1 cells with PP1, a selective inhibitor of Src family kinases, and found that PP1 treatment could attenuate the increased phosphorylation of cortactin induced by MPZL1 overexpression (Figure 7D). These results indicated that the Src kinase activity is essential for MPZL1-mediated migration of $\mathrm{HCC}$ cells. 
Finally, we examined the effect of MPZL1 overexpression on the phosphorylation of cortactin and Src in human HCC tissues by immunoblotting. The results showed that the protein levels of MPZL1 were significantly increased in HCC tissues, while the phosphorylation levels of cortactin and Src were also predominantly increased in HCC tissues compared with the adjacent non-tumor tissues except that the phosphorylation level of Src in case 7 was decreased in HCC tissue (Figure 7E). Together, these results indicated that the MPZL1induced phosphorylation and activation of cortactin is dependent on the activation of Src kinase.

\section{Discussion}

Copy number gain of $1 \mathrm{q}$ is one of the most frequent genetic alterations in primary $\mathrm{HCC}$, detected in 58\% $78 \%$ of HCC patients [14]. Moreover, amplification of 1q has frequently been detected in many other solid tumors, including breast, ovarian, prostate, and lung tumors [3740]. In our previous study, a focal amplified region has been narrowed down to 1q24.1-24.2, suggesting the existence of a cancer gene at this amplicon. Therefore, the identification of the targeted genes responsible for the 1q24.1-24.2 amplification event is imperative for understanding the molecular mechanisms of cancer initiation and progression in many solid tumors including HCC. In the current study, we found that the recurrent region of the 1q24.1-24.2 amplicon specifically targets the MPZL1 gene in HCC.

However, the correlation coefficient $\mathrm{R}$ between the DNA dosage and mRNA expression level of MPZL1 is 0.329 . The result suggests that there is a weak correlation between the DNA dosage and expression levels of MPZL1 gene, which implies that other regulatory mechanisms account for the upregulation of MPZL1 gene expression in HCC, such as DNA methylation. We examined the methylation status of $\mathrm{CpG}$ islands within MPZL1 promoter using quantitative real-time methylation-specific PCR (MSP) on 37 cases of paired HCC and non-tumor tissues. The results showed that the frequency of hypomethylation is approximately $51.4 \%(19 / 37)$ in HCCs, which may account for the upregulation of MPZL1 mRNA level in HCC tissues in addition genomic gain of 1q24.1-24.2 loci (Supplementary information, Figure S8).

MPZL1 was originally isolated as a binding protein and putative substrate of SHP-2. Its extracellular segment has significant sequence similarity to that of myelin $\mathrm{P} 0$, whereas its intracellular portion has two ITIMs that specifically interact with SHP-2 [41]. Moreover, MPZL1 is a highly conserved protein and is expressed in many cell types, suggesting its role in some basic cell functions [20]. However, the biological functions and clinical significance of this gene in human cancers are still elusive. In this study, we first found that there is a positive correlation between the expression levels of MPZL1 and intrahepatic metastasis in HCC specimens. Furthermore, MPZL1 was observed to increase the in vitro migratory and in vivo metastatic potential of HCC cells.

Microarray technologies have been widely used in exploring cancer signaling pathways [42], including gene expression array [43], microRNA array [44] and protein array [45]. Recently, there is an intense interest in applying proteomics to foster an improved understanding of cancer pathogenesis [42]. For example, Kang et al. [27] performed a phospho-proteomics-based study using a phospho-antibody microarray to comprehensively provide mechanistic insight into the role of RSK2 in HNSCC metastasis. To further explore the underlying mechanisms by which MPZL1 stimulates cell migration and tumor metastasis, we used a phospho-antibody microarray-based proteomics approach [27], which has the unique capability of quantitative profiling of protein phosphorylation levels through the use of paired unphospho- and phospho-antibodies for each protein. We identified multiple pro-metastatic proteins of which the phosphorylation and activation levels were regulated by MPZL1 in HCC cells, including ERK-1/2, AKT and cortactin.

Previous study has shown that the phosphorylated MPZL1 mutant (deletion of an extracullular and transmembrane domain) inhibits serum- and growth factorinduced activation of ERK1/2 by blocking the normal function of SHP-2 [46], implicating the roles of MPZL1 in the activation of ERK1/2. In the present study, we also determined the effects of MPZL1 overexpression on the phosphorylation of ERK-1/2 and AKT in HCC cells by immunoblotting. The results showed that MPZL1 overexpression increases the phosphorylation levels of ERK-1/2 (T202/Y204) and AKT (Ser473), which was consistent with the phospho-proteomic array (Supplementary information, Figure S9).

Cortactin, an actin-binding protein and a substrate of Src, is encoded by the CTTN oncogene [47]. Recently, many studies have documented a role for cortactin in promoting cancer cell motility and invasion, including a critical role in invadopodia, which are actin-rich subcellular protrusions associated with the degradation of the extracellular matrix by cancer cells [48, 49]. Notably, orthotopic injection of HCC cells overexpressing cortactin into the liver resulted in an increased intrahepatic metastasis [50]. In the present study, we found that one of the mechanisms by which MPZL1 promoted HCC cell 
migration and tumor metastasis is through the phosphorylation and activation of cortactin. It has been well documented that Src family kinases are the major kinases that promote the tyrosine phosphorylation of cortactin [51]. Moreover, MPZL1 has also been shown to activate Src kinase upon stimulation of cells with extracellular stimuli $[20,34]$. In this study, we found that MPZL1-induced phosphorylation and activation of cortactin is dependent on the activation of Src kinase.

In conclusion, our findings suggested that the MPZL1 is a target gene within the 1q24.1-24.2 amplicon that plays a pivotal role in HCC cell migration and tumor metastasis, and a novel MPZL1/Src/cortactin signaling cascade may be a key component of the MPZL1 prometastatic signaling network in $\mathrm{HCC}$ cells.

\section{Materials and Methods}

\section{Cell lines and cell culture}

A total of seven liver cancer cell lines were used: HepG2, HUH-7, SMMC-7721, Hep3B, SK-HEP-1, HCCLM3 and Li-7. The SMMC-7721 cells were cultured at $37{ }^{\circ} \mathrm{C}$ in a $5 \% \mathrm{CO}_{2}$ atmosphere in DMEM supplemented with $10 \%$ newborn calf serum, $100 \mathrm{U} / \mathrm{ml}$ penicillin and $100 \mu \mathrm{g} / \mathrm{ml}$ streptomycin. The other six cancer cell lines and HEK-293T were cultured in DMEM supplemented with $10 \%$ fetal bovine serum, $100 \mathrm{U} / \mathrm{ml}$ penicillin, and $100 \mu \mathrm{g} / \mathrm{ml}$ streptomycin. The cells were regularly certified as being free of mycoplasma contamination.

\section{Plasmids and other reagents}

The lentiviral shRNA vectors targeting MPZL1 and scrambled control shRNA were purchased from Open Biosystems. The siRNAs targeting CTTN, Src, SHP-2 and the negative control were ordered from GenePharma (Shanghai, China) (Supplementary information, Table S5). In the current study, we employed three siRNAs or shRNAs to knock down the target genes (MPZL1, CTTN, Src and SHP2). Three different shRNAs were used to target the MPZL1 gene, whereas three different siRNAs were used to target the CTTN, Src and SHP2 genes. The knockdown efficiency of each siRNA or shRNA was examined by immnuoblotting. Recently, siRNA pool has been widely used in RNA interference assays [52, 53]. For the $S r c$ and $S H P-2$ genes, we employed siRNA pools (mixture of three different siRNAs) against individual genes, and the results showed that the siRNA pool and individual siRNA can significantly knock down the target genes (Supplementary information, Figure S10). For the CTTN gene, all the three siRNAs can significantly knock down this gene (Supplementary information, Figure S10). Subsequently, the siRNA sequence that was most effective in targeting CTTN (siRNA-CTTN-1) was used to construct shRNA vectors. The Src kinase inhibitor (PP1) was purchased from Sigma.

\section{Lentiviral vector construction, package and infection}

The lentiviral constructs for MPZL1 (isoform a, full-length $M P Z L 1)$ and $C T T N$ were made as described in the previous studies [12]. The entire coding sequence of the target cDNAs was amplified and cloned into the pWPXL vector, which was obtained from Addgene. The phospho-mimetic mutant Y421D (Y421/470/486D) and phospho-deficient mutant Y421A (Y421/470/486A) for cortactin, and a dominant-negative Src construct Src DN (K298M/Y530F) were generated using a twostep PCR-based mutagenesis method as previously described [54]. In brief, the first round of PCR amplified two DNA fragments with overlapping sequences that incorporated mutations. The second round of PCR used these two fragments as templates to generate a full-length mutagenized DNA fragment. Details of the primer sequences and restriction sites are provided in Supplementary information, Table S6. Lentiviral shRNA vectors targeting the CTTN were constructed as described previously [55]. Lentiviral production and transduction were performed according to instructions supplied by Addgene (http://www.addgene.org).

\section{HCC specimens and clinical information}

$\mathrm{HCC}$ and the matched, adjacent non-tumor tissues $(\mathrm{t} 3 \mathrm{~cm}$ from the tumor) were obtained from the surgical specimen archives of the Qidong Liver Cancer Institute, Jiangsu Province, China. Participants from whom these samples were obtained provided their written informed consent to participate in the study, and the Ethical Review Committee of the WHO Collaborating Center for Research in Human Production authorized by the Shanghai Municipal Government approved this study as well as the consent procedure. Genomic DNAs were extracted from 125 HCC primary tumor and adjacent non-tumor tissues, which were subjected to q-PCR analysis of the DNA dosages of MPZL1. Total RNA was extracted from 130 HCC primary tumor and adjacent non-tumor tissues, which were reversely transcribed to a complementary DNA (cDNA) and then subjected to q-PCR analysis of the mRNA expression of MPZL1. The number of overlapping HCC specimens between the $125 \mathrm{HCC}$ specimens with genomic DNA and $130 \mathrm{HCC}$ specimens with cDNA was 46, which were further used to analyze the correlation between DNA dosage and mRNA expression of the MPZL1 gene. Among the 130 paired HCC specimens with cDNA, 58 HCC specimens with clinical and pathological information were used to analyze the correlation between mRNA expression of the MPZL1 gene and clinical features of HCC (Supplementary information, Table S7). The correlations between DNA dosage and mRNA expression of MPZL1 gene were tested using the linear Pearson's R correlation coefficients. The Pearson correlation coefficients were interpreted using the scale provided by Salkin, where an R between 0.8 and 1.0 is defined as very strong, between 0.6 and 0.8 as strong, between 0.4 and 0.6 as moderate, between 0.2 and 0.4 as weak and between 0.0 and 0.2 as very weak or no relationship [56]. In this study, the criteria employed to define the intrahepatic metastasis was as follows: (1) Whether they represent portal-vein tumor thrombi or grew contiguously with vascular thrombi, (2) whether they are small compared to the tumor that they surround, (3) whether a single tumor is present near the main tumor but is much smaller in size and exhibits the same histology [57]. In addition, we mainly employed the Edmondson and Steiner grading system (EGS) to determine the histopathological grade of HCC, and two categories were considered (low grade, EGS I-II; high grade, III-IV) [58].

\section{Quantitative real-time PCR}

Genomic DNA dosage and mRNA expression levels were quantified using a 7900 Real-Time PCR System with SDS 2.3 software (Applied Biosystems), according to the manufacturer's 
instructions. In brief, the total genomic DNA and mRNA were extracted from tumor tissues. First-strand cDNA synthesis and amplification were performed using Reverse Transcription Reagents (Takara) per the manufacturer's instructions. Briefly, cDNA templates were combined with SYBR Green premix with Rox (Takara) for quantitative PCR reactions. A repetitive element (LINE-1) and $\beta$-actin were used as the endogenous controls for the DNA and mRNA levels, respectively. DNA dosage was normalized to LINE-1 and gene expression was normalized to $\beta$-actin. DNA content was normalized to that of LINE-1, a repetitive element whose copy numbers per haploid genome are similar among all human cells (normal or neoplastic) [59]. Details of the primers sequences are provided in Supplementary information, Table S6. The human MPZL1 gene has three isoforms (MPZL1, MPZL1a and MPZL1b), all of which were subjected to q-PCR analysis for the relative expression levels of each isoform in 58 pairs of $\mathrm{HCC}$ and adjacent non-tumor tissues.

\section{Quantitative real-time MSP}

The experiments were performed as previously described with some modifications [60]. In brief, two real-time MSP systems were developed for the detection and quantitation of the bisulfiteconverted methylated version of the MPZL1 gene (the MPZL1$M$ system; system 1) and the bisulfite-converted unmethylated version of the MPZL1 gene (the MPZL1-U system; system 2). For system 1, the primers MPZL1-MF (5'-GTAAAGGATAGGTTTTGGGGATAC-3') and MPZL1-MR (5'-TCAACTCTTCTTATTCTCTACCGAC-3') were used. For system 2, the primers MPZL1-UF (5'-TAAAGGATAGGTTTTGGGGATATG-3') and MPZL1-UR (5'-TCAACTCTTCTTATTCTCTACCAAC-3') were used. The DNA templates were combined with SYBR Green premix with Rox (Takara) for quantitative-PCR reactions. $\beta$-actin was used as the endogenous control for DNA levels.

\section{Cell proliferation assays}

Cells were seeded at a density of 2000 cells per well in 96well plates and incubated. An aliquot of $10 \mu \mathrm{l}$ of Cell-Counting Kit (CCK-8, Dojindo, Kumamoto, Japan) was added to the wells and incubated for $2 \mathrm{~h}$. The absorbance was measured at 450 $\mathrm{nm}$ to calculate the numbers of viable cells in each well. Each measurement was performed in triplicate and the experiments were repeated twice.

For colony formation assays, cells were seeded in 6-well plates at a density of 200 cells per well and cultured at $37{ }^{\circ} \mathrm{C}$ for 2 weeks. At the end of the incubation, the cells were fixed with $100 \%$ methanol and stained with $0.1 \%$ (W/V) Crystal violet. Megascopic cell colonies were counted using Image-Pro Plus 5.0 software (Media Cybernetics, Bethesda, MD, USA). Each measurement was performed in triplicate and the experiments were each conducted at least three times.

\section{Transwell migration and invasion assays}

Cell migration and invasion assays were performed using 6.5$\mathrm{mm}$ transwell chambers $(8-\mu \mathrm{m}$ pore size, $\mathrm{BD})$ as described previously, with certain modifications [55]. Cells were seeded at a density of 40000 and 80000 cells per well into transwell chambers for migration and invasion assays, respectively. The wells were washed with PBS after $16 \mathrm{~h}$ for HUH-7, SMMC-7721 and Li-7 cells and $4 \mathrm{~h}$ for SK-HEP-1 cells. The cells that had migrated to the basal side of the membrane were fixed and stained with Crystal violet, visualized and photographed with a CKX41 microscope (Olympus, Japan) at $200 \times$ magnification. Images of three random fields from three replicate wells were obtained, and the cells that had migrated were counted.

\section{Animal experiments and histological analysis}

Animal experiments were performed as described previously [55]. Briefly, for primary tumor growth assays, cells $\left(2 \times 10^{6}\right.$ per mouse) were injected subcutaneously into the right upper flank region of the nude mice. The mice were monitored weekly for tumor size and evidence of morbidity. The tumor size was quantified in two dimensions using calipers. Tumor volume was calculated as follows: tumor volume $\left(\mathrm{mm}^{3}\right)=\mathrm{L} \times \mathrm{W} \times \mathrm{W} / 2$, where $\mathrm{L}$ represents length and $\mathrm{W}$ represents width.

For in vivo metastasis assays, cells $\left(2 \times 10^{6}\right.$ per mouse $)$ were injected into the tail veins of nude mice. Six weeks later, all of the mice were euthanized and the organs, including the lungs and livers, were removed and processed for standard histological studies. For histological analysis, the primary tumors and mouse organs were harvested at necropsy and fixed in $10 \%$ formalin. The fixed samples were then embedded in paraffin, and three non-sequential serial sections were obtained from each animal. The sections were stained with hematoxylin and eosin (H\&E) and analyzed for the presence of metastases.

\section{Phosphory-antibody array}

The experiment was performed as described previously [27]. Cell lysates obtained from HUH-7 VECTOR and HUH-7 MPZL1 cells were applied to the Phospho Explorer Antibody Array (PEX100), which was designed and manufactured by Full Moon Biosystems Inc.

\section{Src inhibitor treatments}

For inhibitor treatments, cells were plated at a density such that they would reach $80 \%$ confluency on the following day. Cells were treated with DMSO or PP1 at different concentrations $(0.1,0.17$ and $1 \mu \mathrm{M})$ in the complete medium. After $24 \mathrm{~h}$, cells were harvested for western blot or transwell migration assays.

\section{Western blot analysis}

For western blot analysis, cells were lysed and the protein concentration was determined by the Bradford assay. The cell lysates were separated by SDS-PAGE and transferred to polyvinylidene difluoride membranes. The membranes were blocked and incubated with specific primary antibodies. Specific antibodies against MPZL1, phospho-MPZL1 (Y263), cortactin, phospho-cortactin (Y421), FAK, phospho-FAK (Y397), SHP-2, phospho-SHP-2 (Y580), Src and phospho-Src (Y416) were from Cell Signaling Technology; the antibody against $\beta$-actin was purchased from Sigma.

\section{Statistical analysis}

The statistical analysis and graphical presentation were performed using GraphPad Prism 5.0. The results are presented as the mean \pm s.e.m and were evaluated with unpaired Student's $t$-test (two-tailed; $P<0.05$ was considered significant), unless specified otherwise (paired $t$-test, the Pearson's correlation and one-way ANOVA analysis). Specifically, statistical analysis of differences 
between groups was performed by unpaired Student's $t$-test and $P$ $<0.05$ was considered statistically significant. A paired Student's $t$-test was used to analyze differences in mRNA expression levels among tumors and paired non-tumor tissues in q-PCR analysis. In addition, certain statistical calculations were performed using SPSS (Statistical Package for the Social Sciences), version 19.0 for Windows. The $\chi^{2}$ test was used to evaluate the association between MPZL1 expression and the clinicopathological parameters of the HCC specimens. $P$ values $<0.05$ were considered significant.

\section{Acknowledgments}

We are very grateful to professor Didier Trono (Ecole Polytechnique Fédérale de Lausanne, 1015 Lausanne, Switzerland) for providing the pWPXL, psPAX2 and pMD2.G lentivirus plasmids . This work was partially supported by the National Key Basic Research Program of China (2013CB910500), the National Natural Science Foundation of China (81125016, 81071637 and 91029728), Shanghai Science and Technology Commission (11XD1404500), Shanghai Municipal Education Commission and Shanghai Municipal Health Bureau (11SG18 and XBR2011039).

\section{References}

1 Lau SH, Sham JS, Xie D, et al. Clusterin plays an important role in hepatocellular carcinoma metastasis. Oncogene 2006; 25:1242-1250.

2 Yang JM, Peng ZH, Si SH, Liu WW, Luo YH, Ye ZY. KAI1 gene suppresses invasion and metastasis of hepatocellular carcinoma MHCC97-H cells in vitro and in animal models. Liver Int 2008; 28:132-139.

3 Wong CC, Wong CM, Tung EK, Man K, Ng IO. Rho-kinase 2 is frequently overexpressed in hepatocellular carcinoma and involved in tumor invasion. Hepatology 2009; 49:1583-1594.

4 Lee TK, Poon RT, Yuen AP et al. Twist overexpression correlates with hepatocellular carcinoma metastasis through induction of epithelial-mesenchymal transition. Clin Cancer Res 2006; 12:5369-5376.

5 Yao J, Liang L, Huang S et al. MicroRNA-30d promotes tumor invasion and metastasis by targeting Galphai2 in hepatocellular carcinoma. Hepatology 2010; 51:846-856.

6 Ding J, Huang S, Wu S et al. Gain of miR-151 on chromosome $8 \mathrm{q} 24.3$ facilitates tumour cell migration and spreading through downregulating RhoGDIA. Nat Cell Biol 2010; 12:390-399.

7 Ying Q, Liang L, Guo W et al. Hypoxia-inducible microRNA-210 augments the metastatic potential of tumor cells by targeting vacuole membrane protein 1 in hepatocellular carcinoma. Hepatology 2011; 54:2064-2075.

8 Whittaker S, Marais R, Zhu AX. The role of signaling pathways in the development and treatment of hepatocellular carcinoma. Oncogene 2010; 29:4989-5005.

9 Shlien A, Malkin D. Copy number variations and cancer susceptibility. Curr Opin Oncol 2010; 22:55-63.

10 Hahn WC, Dunn IF, Kim SY et al. Integrative genomic approaches to understanding cancer. Biochim Biophys Acta 2009; 1790:478-484.

11 Firestein R, Bass AJ, Kim SY et al. CDK8 is a colorectal cancer oncogene that regulates beta-catenin activity. Nature 2008; 455:547-551.
12 Jia D, Wei L, Guo W et al. Genome-wide copy number analyses identified novel cancer genes in hepatocellular carcinoma. Hepatology 2011; 54:1227-1236.

13 Sawey ET, Chanrion M, Cai C et al. Identification of a therapeutic strategy targeting amplified FGF19 in liver cancer by Oncogenomic screening. Cancer Cell 2011; 19:347-358.

14 Lau SH, Guan XY. Cytogenetic and molecular genetic alterations in hepatocellular carcinoma. Acta Pharmacol Sin 2005; 26:659-665.

15 Chen L, Chan TH, Guan XY. Chromosome 1q21 amplification and oncogenes in hepatocellular carcinoma. Acta Pharmacol Sin 2010; 31:1165-1171.

$16 \mathrm{Ma}$ NF, Hu L, Fung JM et al. Isolation and characterization of a novel oncogene, amplified in liver cancer 1, within a commonly amplified region at 1q21 in hepatocellular carcinoma. Hepatology 2008; 47:503-510.

17 Zannettino AC, Roubelakis M, Welldon KJ et al. Novel mesenchymal and haematopoietic cell isoforms of the SHP-2 docking receptor, PZR: identification, molecular cloning and effects on cell migration. Biochem J 2003; 370:537-549.

18 Roubelakis MG, Martin-Rendon E, Tsaknakis G, Stavropoulos A, Watt SM. The murine ortholog of the SHP-2 binding molecule, PZR accelerates cell migration on fibronectin and is expressed in early embryo formation. J Cell Biochem 2007; 102:955-969.

19 Eminaga S, Bennett AM. Noonan syndrome-associated SHP2/Ptpn11 mutants enhance SIRPalpha and PZR tyrosyl phosphorylation and promote adhesion-mediated ERK activation. J Biol Chem 2008; 283:15328-15338.

20 Kusano K, Thomas TN, Fujiwara K. Phosphorylation and localization of protein-zero related (PZR) in cultured endothelial cells. Endothelium 2008; 15:127-136.

21 Roessler S, Jia HL, Budhu A et al. A unique metastasis gene signature enables prediction of tumor relapse in earlystage hepatocellular carcinoma patients. Cancer Res 2010; 70:10202-10212.

22 Chen X, Cheung ST, So S et al. Gene expression patterns in human liver cancers. Mol Biol Cell 2002; 13:1929-1939.

23 Cui JF, Liu YK, Zhang LJ et al. Identification of metastasis candidate proteins among HCC cell lines by comparative proteome and biological function analysis of S100A4 in metastasis in vitro. Proteomics 2006; 6:5953-5961.

24 Genda T, Sakamoto M, Ichida T et al. Cell motility mediated by rho and Rho-associated protein kinase plays a critical role in intrahepatic metastasis of human hepatocellular carcinoma. Hepatology 1999; 30:1027-1036.

25 Anderson K, Potter A, Baban D, Davies KE. Protein expression changes in spinal muscular atrophy revealed with a novel antibody array technology. Brain 2003; 126:2052-2064.

26 Huang GJ, Yang CM, Chang YS et al. Hispolon suppresses SK-Hep1 human hepatoma cell metastasis by inhibiting matrix metalloproteinase-2/9 and urokinase-plasminogen activator through the PI3K/Akt and ERK signaling pathways. $J$ Agric Food Chem 2010; 58:9468-9475.

27 Kang S, Elf S, Lythgoe K et al. p90 ribosomal S6 kinase 2 promotes invasion and metastasis of human head and neck squamous cell carcinoma cells. J Clin Invest 2010; 120:11651177.

28 von Sengbusch A, Gassmann P, Fisch KM, Enns A, Nicolson 
GL, Haier J. Focal adhesion kinase regulates metastatic adhesion of carcinoma cells within liver sinusoids. Am J Pathol 2005; 166:585-596.

29 Summy JM, Gallick GE. Src family kinases in tumor progression and metastasis. Cancer Metastasis Rev 2003; 22:337358.

30 Buday L, Downward J. Roles of cortactin in tumor pathogenesis. Biochim Biophys Acta 2007; 1775:263-273.

31 Yamada S, Yanamoto S, Kawasaki G, Mizuno A, Nemoto TK. Overexpression of cortactin increases invasion potential in oral squamous cell carcinoma. Pathol Oncol Res 2010; 16:523-531.

32 Li Y, Tondravi M, Liu J et al. Cortactin potentiates bone metastasis of breast cancer cells. Cancer Res 2001; 61:69066911.

33 Kanner SB, Reynolds AB, Vines RR, Parsons JT. Monoclonal antibodies to individual tyrosine-phosphorylated protein substrates of oncogene-encoded tyrosine kinases. Proc Natl Acad Sci U S A 1990; 87:3328-3332.

34 Zhao R, Guerrah A, Tang H, Zhao ZJ. Cell surface glycoprotein PZR is a major mediator of concanavalin A-induced cell signaling. J Biol Chem 2002; 277:7882-7888.

35 Walter AO, Peng ZY, Cartwright CA. The Shp-2 tyrosine phosphatase activates the Src tyrosine kinase by a non-enzymatic mechanism. Oncogene 1999; 18:1911-1920.

36 Luttrell LM, Hawes BE, van Biesen T, Luttrell DK, Lansing TJ, Lefkowitz RJ. Role of c-Src tyrosine kinase in G proteincoupled receptor- and Gbetagamma subunit-mediated activation of mitogen-activated protein kinases. J Biol Chem 1996; 271:19443-19450.

37 Shadeo A, Lam WL. Comprehensive copy number profiles of breast cancer cell model genomes. Breast Cancer Res 2006; 8:R9.

38 Partheen K, Levan K, Osterberg L, Helou K, Horvath G. Analysis of cytogenetic alterations in stage III serous ovarian adenocarcinoma reveals a heterogeneous group regarding survival, surgical outcome, and substage. Genes Chromosomes Cancer 2004; 40:342-348.

39 Saramaki OR, Porkka KP, Vessella RL, Visakorpi T. Genetic aberrations in prostate cancer by microarray analysis. Int $J$ Cancer 2006; 119:1322-1329.

40 Weir BA, Woo MS, Getz G et al. Characterizing the cancer genome in lung adenocarcinoma. Nature 2007; 450:893-898.

41 Zhao ZJ, Zhao R. Purification and cloning of PZR, a binding protein and putative physiological substrate of tyrosine phosphatase SHP-2. J Biol Chem 1998; 273:29367-29372.

42 Sanchez-Carbayo M. Antibody arrays: technical considerations and clinical applications in cancer. Clin Chem 2006; 52:16511659.

43 Roberts CJ, Nelson B, Marton MJ et al. Signaling and circuitry of multiple MAPK pathways revealed by a matrix of global gene expression profiles. Science 2000; 287:873-880.

44 Webster RJ, Giles KM, Price KJ, Zhang PM, Mattick JS, Leedman PJ. Regulation of epidermal growth factor receptor signaling in human cancer cells by microRNA-7. J Biol Chem
2009; 284:5731-5741.

45 Rikova K, Guo A, Zeng Q et al. Global survey of phosphotyrosine signaling identifies oncogenic kinases in lung cancer. Cell 2007; 131:1190-1203.

46 Zhao R, Fu X, Teng L, Li Q, Zhao ZJ. Blocking the function of tyrosine phosphatase SHP-2 by targeting its Src homology 2 domains. $J$ Biol Chem 2003; 278:42893-42898.

47 Chen L, Wang ZW, Zhu JW, Zhan X. Roles of cortactin, an actin polymerization mediator, in cell endocytosis. Acta Biochim Biophys Sin (Shanghai) 2006; 38:95-103.

48 Sung BH, Zhu X, Kaverina I, Weaver AM. Cortactin controls cell motility and lamellipodial dynamics by regulating ECM secretion. Curr Biol 2011; 21:1460-1469.

49 Eckert MA, Lwin TM, Chang AT et al. Twist1-induced invadopodia formation promotes tumor metastasis. Cancer Cell 2011; 19:372-386.

50 Chuma M, Sakamoto M, Yasuda J et al. Overexpression of cortactin is involved in motility and metastasis of hepatocellular carcinoma. J Hepatol 2004; 41:629-636.

51 Weaver AM. Cortactin in tumor invasiveness. Cancer Lett 2008; 265:157-166.

52 Echeverri CJ, Perrimon N. High-throughput RNAi screening in cultured cells: a user's guide. Nat Rev Genet 2006; 7:373384.

53 Whitehurst AW, Bodemann BO, Cardenas J et al. Synthetic lethal screen identification of chemosensitizer loci in cancer cells. Nature 2007; 446:815-819.

54 Ren G, Helwani FM, Verma S, McLachlan RW, Weed SA, Yap AS. Cortactin is a functional target of E-cadherin-activated Src family kinases in MCF7 epithelial monolayers. $J$ Biol Chem 2009; 284:18913-18922.

55 Jia D, Yan M, Wang X et al. Development of a highly metastatic model that reveals a crucial role of fibronectin in lung cancer cell migration and invasion. BMC Cancer 2010; 10:364.

56 Perek B, Malinska A, Stefaniak S et al. Predictive factors of late venous aortocoronary graft failure: ultrastructural studies. PLoS One 2013; 8:e70628.

57 Sakamoto M, Hirohashi S, Tsuda H, Shimosato Y, Makuuchi M, Hosoda Y. Multicentric independent development of hepatocellular carcinoma revealed by analysis of hepatitis B virus integration pattern. Am J Surg Pathol 1989; 13:1064-1067.

58 Pirisi M, Leutner M, Pinato DJ et al. Reliability and reproducibility of the edmondson grading of hepatocellular carcinoma using paired core biopsy and surgical resection specimens. Arch Pathol Lab Med 2010; 134:1818-1822.

59 Zhao X, Li C, Paez JG et al. An integrated view of copy number and allelic alterations in the cancer genome using single nucleotide polymorphism arrays. Cancer Res 2004; 64:30603071.

60 Fackler MJ, McVeigh M, Mehrotra J, et al. Quantitative multiplex methylation-specific PCR assay for the detection of promoter hypermethylation in multiple genes in breast cancer. Cancer Res 2004; 64:4442-4452.

(Supplementary information is linked to the online version of the paper on the Cell Research website.) 